\title{
Estimated Vestibulogram (EVEST): Effective Graphic Tool for Vestibular Assessment
}

\author{
Maja Striteska ${ }^{1}$, Lukáš Školoudík ${ }^{2}$, Martin Chovanec $^{3}$, Oliver Profant ${ }^{3}$, Jan Mejzlik ${ }^{4}$, \\ Viktor Chrobok ${ }^{1}$, Katerina Trnkova ${ }^{3}$, Martin Valis ${ }^{1}$, Michael Maslin ${ }^{5}$, and Jan Kremlacek ${ }^{6}$ \\ ${ }^{1}$ University Hospital Hradec Kralove \\ ${ }^{2}$ Fakultni Nemocnice Hradec Kralove \\ ${ }^{3}$ University Hospital Kralovske Vinohrady \\ ${ }^{4}$ University Hospital Hradec Kralove, Charles University in Prague, Faculty of Medicine in \\ Hradec Kralove \\ ${ }^{5}$ Interacoustics A/S \\ ${ }^{6}$ Charles University Faculty of Medicine in Hradec Kralove
}

May 11, 2020

\begin{abstract}
BACKGROUND: The availability and development of the methods testing the vestibuloocular reflex (VOR) bring a broader view into the lateral semi-circular canal (L-SCC) function. At the same time, a higher number of evaluated parameters increases demands on the specialist's diagnose-making process. OBJECTIVE: To provide medical specialists a new diagnostic-graphic tool, Estimated Vestibulogram- EVEST, enabling a quick and easy-to-read visualization of the enhanced VOR test results within the L-SCC. METHODS: The research data for the development of the EVEST involved 148 participants including 49 healthy volunteers ( 28 female and 21 male) and 99 (58 female and 41 male) patients with a diverse degree of peripheral vestibular deficit. The correspoding L-SCC VOR test results, from patients meeting the diagnostic criteria, were used to create the EVEST. RESULTS: Based on the test results from the sample of 148 participants we demonstrate on cumulative and single EVEST graphs that the EVEST is a functional and reliable tool for quick graphic diagnosis of diverse types and levels of the peripheral vestibular loss. CONCLUSIONS: Unique patterns of the EVEST can help to easily distinguish various types and levels of the peripheral vestibular loss.
\end{abstract}

\section{Keywords}

Estimated Vestibulogram, EVEST, Lateral Semi-circular Canal, Vestibuloocular Reflex, Caloric Test, Videooculography, Spontaneous Nystagmus, Head Shaking Test, Vestibular Evoked Myogenic Potentials, Video Head Impulse Test

\section{Abbreviations}

average Slow Phase Velocity (aSPV), Directional Preponderance (DP), Estimated Vestibulogram (EVEST), Head Shaking Test (HST), horizontal Vestibuloocular Reflex (hVOR), Lateral Semi-circular Canal (L-SCC), Monothermal Caloric Warm Screening Test (MWST), Spontaneous Nystagmus (SN), , Total Response (TR), unilateral weakness (UW), Vestibular Evoked Myogenic Potentials (VEMP), Vestibuloocular reflex (VOR), Videooculography (VOG), Video Head Impulse Test (VHIT)

\section{Introduction}


Recently, the otoneurology has undergone a major change due to improvement of testing instruments with the video eye tracking systems. Eye movements and nystagmus can be nowadays observed with extremely high sensitivity[1]. The eye tracking system devices are mainly focused on testing the efficiency of theVestibuloocular reflex (VOR). Although various vestibular tests have brought a more complex insight into the vestibular pathophysiology, the same situation also increased demands on medical professional's ability to interpret the complex of data. A higher number of evaluated parameters is needed to establisha correct diagnosis.

Clinicians compare all the data and search for their interrelations as well as for synoptic context, which is often difficult to analyze. In order to assist clinicians in complex data analysis we have developed a new graphic tool, which we call an Estimated Vestibulogram (EVEST). The main aim of the EVEST is to provide quick and easy-to-read overview of different vestibular test results,all in "one place". EVEST was constructed tovisualize the VOR efficiency and frequency-specific changes of the lateral semicircular canal function (L-SCC).

In our study we test the competence of the EVEST to visualize different vestibular lesions. Also, we test EVEST's ability to show each test sensitivity to different vestibular diagnoses in the cumulative EVEST graphs, since different diagnoses can manifest with various VOR frequency damage. The aim of our study was to assess the effectiveness of new graphic tool called EVEST and its reliability.

\section{Methods}

\subsection{Participants}

Data from 148 participants were used to construct and test the feasibility of the EVEST. We examined 49 healthy volunteers (28 female and 21 male) and 99 patients(58 female and 41 male)with various degree of unilateral peripheral vestibular deficit divided into two groups, vestibular neuritis and vestibular schwannoma group.The inclusion criterion for the vestibular neuritis group wasunilateral peripheral vestibular lesion without any neurological nor cochlear deficit.The inclusion criterion for the schwannoma group wasanMRI confirmed diagnosis of the vestibular schwannoma. Participants were examined from January 2016 to July 2019 in University Hospital Hradec Kraloveand University Hospital Kralovske Vinohrady, Prague, Czech Republic. All participants underwent complex neuro-otologic examinationperformed by VOGdevice (VisualEyes 525, Interacoustics) and VHITdevice (EyeSeeCam vHIT, Interacoustics).

\subsection{Ethical considerations}

(removed for blind peer review)

\subsection{Test battery}

Full test battery of VOG included Spontaneous Nystagmus recording (SPN), Head Shaking Test (HST), Gaze Evoked Nystagmus recording, Smooth Pursuit, Saccadic Eye Movements, Optokinetic Nystagmus, Positional and Positioning Tests, Air Conducted Bithermal Caloric Test, followed by Cervical and Ocular Vestibular Evoked Myogenic Potentials, Subjective Visual Vertical, and Video Head Impulse Test (VHIT). Further on, to contribute to the construction of the EVEST, the following results of horizontal vestibuloocular reflex (hVOR) tests were used: average slow-phase velocities (aSPV) of spontaneous and/or head-shaking evoked nystagmus; unilateral weakness (UW), directional preponderance or total caloric response values based on bithermal caloric stimulation (air conducted); and VHIT gains and side difference. In order to be able to plot the results into the EVEST, it was necessary to use eye tracker devices- VOG (prior to ENG) and VHIT.

\subsubsection{SPN recording method}

The patient is instructed to sit down and maintain his head in the primary position with a slight inclination of the head, and to look straight ahead, the visual fixation denied (goggle closure). Tracing for 60 seconds is recorded. If nystagmus appears, short visual fixation period of approximately 5-10 seconds with a light spot is recorded. If any ambiguity regarding technical quality of the tracing or about the elucidated eye movements is present, the test is repeated,until the true abnormalities are consistent and repeatable (Bahrin, ASHA). There is a debate in the literature, and the evidence supporting or rejecting the use of mental alerting [2-4]. 
Based on our experience, the mental alerting is not recommended. From our evidence, any mental alerting results in inadequate eye movements, which increases the noise. Further we recommend a quiet zone for the SPN examination. After recording is done, we should verify that any nystagmus has been correctly identified by the equipment's software and edit, if necessary. If any nystagmus occurs, we should establish its slow component velocity and duration.

\subsubsection{VOG assisted bithermal air caloric test procedure}

The patient lies down in a caloric position with the head 30 degree above supine. We use warm $\left(48^{\circ} \mathrm{C}\right)$ and cold $\left(24^{\circ} \mathrm{C}\right)$ air for 60 seconds, each ear separately, fixation denied, 6 minutes waiting between each irrigation.

\subsubsection{Head Shaking Test (HST) procedure}

The patient is sitting upright, visual fixation denied. Eye movements are observed for a time period of 10 seconds to obtain a baseline. The examiner grasps the patient's head and moves it briskly to left and right (around the vertical axis), aiming for a frequency of about $2 \mathrm{~Hz}$ and a displacement of the head of approximately 20-30 degrees (preferably with the head pitched forward $30^{*}$ to align the horizontal semicircular canals with the plane of head rotation). The head-shaking is continued for 20 cycles and then abruptly stopped. VOG recording for a time period of 120 seconds is done, evoked nystagmic eye movements are evaluated.

\subsubsection{VHIT procedure}

The patient is sitting upright, with visual fixation of the spot in 1-2 $\mathrm{m}$ distance, the examiner grasps the patient's head and moves it briskly and unpredictably for the patient. Peak head velocity of the impulses should be 150deg to $250 \mathrm{deg} / \mathrm{second}$, amplitude of head movement 10deg-20deg.

\subsubsection{Devices used for the study included:}

VOG VisualEyes 525 from Interacoustics, 2D-VOGfw goggle w. side mounted cameras FireWire(r) camera module (two modules in binocular configuration) Capture Resolution: 640 x 480 Pixels @ 100 fps. Video Resolution: 640 x 240 Pixels @ 25 fps Images: 100 frames per second. Horizontal and Vertical Eye movement measurement resolution: approx. 0.33 degrees. Single IR LED infrared illumination: $950 \mathrm{~nm}$ at $1.5 \mathrm{mw} / \mathrm{cm}^{2}$.

VHIT EyeSeeCam Interacoustics, Monocular camera, interchangeable between left and right eye, Sample rate $220 \mathrm{~Hz}$, Instantaneous gains at 40,60 and $80 \mathrm{~ms}$ - equivalent to scleral search coil method. Data Analysis: Velocity Regression Plot, Gain Plot.

\subsection{EVEST basic principles}

The main aim of the EVEST is to simply visualize the results of the different hVOR (horizontal vestibuloocular reflex) vestibular tests (empty EVEST- see Figure 1). EVEST has some similarities to pure tone audiogram which make graph easy to read for otolaryngologists, especially frequency range on the $\mathrm{X}$ axis and functional loss on the $\mathrm{Y}$ axis.Different vestibular tests are placed on the $\mathrm{X}$ axis, according to their tested frequency of head movement. On the $\mathrm{Y}$ axis, there is an approximative vestibular weakness/asymmetry expressed as a percentage loss $0-100 \%$.

To create the EVEST, we used four tests of the hVOR, which together can test a wide frequency range of the hVOR to determine the different vestibular disorders more accurately. The spontaneous nystagmus (SPN) represents the only static test of hVOR in the EVEST, the caloric test (CT), head shaking test (HST) and VHIT are hVOR dynamic tests. These provide complementary insights into the hVOR function.

Vestibular tests on the $\mathrm{X}$ axis represent different head/endolymph movement frequencies. The SPN equals a $0 \mathrm{~Hz}$ frequency of a head/endolymph movement, the caloric test equals the frequency of $0.003-0.004 \mathrm{~Hz}$, HST of $2 \mathrm{~Hz}$, and VHIT of $3-5 \mathrm{~Hz}$. Frequency of each test will be explained further.

\section{Results}

\subsection{Vestibular neuritis group}


To evaluate the feasibility of EVEST we present our vestibular neuritis (VN) group data. Forty-nine patients with VN were included in the study: 30 females and 19 males (mean age 49, SD 13, min 25, max 79) who met the diagnostic criterion of the acute unilateral vestibular loss without cochlear or neurological deficit. Within the first three days of the vertigo onset, SPN, HST and VHIT recordings were performed in all the patients. Only 4 patients had also caloric test during this first period. The majority of the patients underwent the caloric test between $4^{\text {th }}$ to $21^{\text {th }}$ day of the vertigo onset. Mean spontaneous nystagmus (SPN) velocity during the first period was $14 \mathrm{deg} / \mathrm{s}$ aSPV (SD 5.7, min 5,max 31), HST strengthened SPN in $92 \%$ (mean aSPV $17.2 \mathrm{deg} / \mathrm{s}, \mathrm{SD} 5.3, \min 9, \max 31$ ) and only in 4 patients the HST evoked the same or lower nystagmus aSPV than SPN. The mean VHIT gain on the affected side was 0.35 (SD 0.11, min 0.14, $\max$ 0.61 ); on the unaffected side it was 0.84 (SD 0.11, min 0.55, max 1.07) in the acute stage. The caloric test within the first three weeks showed mean unilateral weakness (UW) $76.6 \%$ on the affected side (SD 24, min 4 , $\max 100)$. There were 4 results from the first period $\left(0-3^{\text {rd }}\right.$ day), with false negative low UW results of $4-12 \%$. The false negative values for the unilateral weakness were, in these four patients, subsided by the DP results, which in this particular period, represented the ongoing pathology, and later, on follow-ups were confirmed by pathological unilateral weakness results.

The cumulative EVEST for the vestibular neuritis group is presented in Figure 2, for an example of a single EVEST see Figure 3.

\subsection{Vestibular schwannoma group}

Fifty patients were included in our study; 28 females and 22 males (mean age 54, SD 15, min 15, max 79). The size of the VS was evaluated based on the Koos classification [5]: 13 patients with Koos 1 stage, 21 patients Koos 2, 10 patients Koos 3, and 6 patients Koos 4. The EVEST showed a slowly progressing vestibular deficit especially in caloric test.Mean caloric unilateral weakness on the affected side was $49.8 \%$ (SD 32.3, min 0, $\max 100$ ). Mean SPN slow phase velocity was $0.28 \mathrm{deg} / \mathrm{s}$ aSPV (SD 0.536, min 0, $\max 2$ ), mean HST was1.76deg/s aSPV (SD 2.46, min 0, max 13), mean VHIT gain on the affected side was 0.74 (SD 0.21, min 0.33, max 1.04).Cumulative EVEST for vestibular schwannoma group is presented in Figure 4 , for examples of a single EVEST see Figure 5,6.

\subsection{Control group}

Forty-nine healthy participants were included in our study. The inclusion criterion for healthy volunteers was no evidence of any instability nor vertigo in their previous history. There were 28 female and 21 male volunteers (mean age 40.8, SD 10.4, min 21, max 62). In this group, in sitting and up-right position, no nystagmus was recorded. No head shaking nystagmus was evoked. Unilateral weakness in caloric test was in almost all subjects within a normal range $0-25 \% \mathrm{UW}$ (mean12.7\%, SD 7.3, min 0, max 36). One healthy volunteer showed caloric UW of $36 \%$, but with all the remaining tests within a normal range, and MRI was normal. In 5 healthy volunteers the total caloric response was abnormally low, below the $10 \mathrm{deg} / \mathrm{s}$. Mean VHIT gain was 0.87 (SD 0.04, min 0.78, max 0.98). Cumulative EVEST for the controll group is presented in Figure 7 .

\section{Discussion}

Before we will start to plot the results from our groups into the EVEST, we will clarify the terminology and the plotting principles.

\subsection{Explanation of frequency versus acceleration}

The EVEST is created for VORfrequency range from $0 \mathrm{~Hz}$ to approx. $5 \mathrm{~Hz}$. The term "frequency" (equivalent for a head cyclic changes per second) is used to describe the dynamic stimulus. There is a frequencyrange over which hVOR is optimally stimulated $(0.05-8 \mathrm{~Hz})[6]$.In each dynamic hVOR test, frequency and acceleration correlate in different ways.In a head still position (SPN tracing), observed VOR frequency is $0 \mathrm{~Hz}$, since no head or endolymph movement is present. 
The caloric test is based on the stimulation of the L-SCC by air conducted temperature gradient which deflects the cupula by accelerated endolymphatic flow. One response (one ear, warm or cold) can be taken to represent a half-cycle of a sinusoidal oscillation (since a full oscillation would repeat the process in reverse). Thus, despite varying estimates, typical nystagmus duration is 120 - 180 seconds in healthy individuals. Therefore, the period of a full oscillation would be 240 to 360 seconds, the reciprocal of which is 0.004 to $0.003 \mathrm{~Hz}$. The gained velocity of endolymphatic fluid is not known in any caloric test. However, it can be derived from the output velocity (the slow phase of the caloric evoked nystagmus) because the two should match. During a sinusoidal rotation, the peak slow phase eye velocity is reached at $1 / 4$ of a full oscillationcycle; 60 seconds for a standard water caloric and 80 seconds for an ice cold caloric stimulation[7]. We used the air bithermal caloric test $(24 / 48 \mathrm{deg} C)$. In our healthy group,the average time response was $150 \mathrm{~s}$, therefore $0.003 \mathrm{~Hz}$, with the average peak slow phase velocity of evoked nystagmus at 20-30deg/sec (cold-warm), the peak response was reached in $60-70 \mathrm{sec}$, so the average acceleration was $0.42 \mathrm{deg} / \mathrm{sec}^{2}$ for warm air caloric test, $0.35 \mathrm{deg} / \mathrm{sec}^{2}$ for cold air.

In the case of the HST and VHIT, the acceleration force necessary to stimulate the cupula is provided by passive head rotations. During the head shaking test,one sinusoidal cycle (2cycles per second) takes $0.5 \mathrm{~s}$, with the amplitude of head movement 30deg, and peak velocity of head movement to one side 120-150deg/s, so the acceleration is approx. $960-1,200 \mathrm{deg} / \mathrm{sec}^{2}$.

VHIT peak input velocity varies between $150 \mathrm{deg} / \mathrm{sec}$ and $250 \mathrm{deg} / \mathrm{sec}$ for horizontal head impulses.Despite varying impulse velocities, the period over which the response analysis occurs is fixed. The impulse duration to rotate the head (by approx. 15deg to the left or right) is short, typically several hundred milliseconds. A half-cycle of VHIT head movement (from $0^{\circ} / \mathrm{s}$ to peak of head velocity) lasts for 120-150 ms, so the frequency can vary among skilled examiners. At our department, this interval varies from 120-150 ms. Accordingly, the frequency can vary from 3 to $4 \mathrm{~Hz}$. The calculated acceleration increases from $2,667 \mathrm{deg} / \mathrm{sec}^{2}$ up to $4,167 \mathrm{deg} / \mathrm{sec}^{2}$.

To recap, the caloric test is often described as a low frequency hVOR test, the rotatory chair and HST are described as intermediate and mediate frequency range hVOR tests, and the vHIT as a test with a high frequency stimulation (covering the normal physiological range, where the angular acceleration of a head impulse up to 4,000deg $/ \mathrm{s}^{2}$ occurs during the normal head movements)[8]. Although not often stated explicitly, in this context rotational frequency (or just "frequency") is being used as a synonym for acceleration, or stimulus strength. The stimulus parameter of direct relevance to each hVOR test is an acceleration. Greater acceleration causes greater cupula deflection. The caloric test stimulus provides the weakest acceleration, the rotatory chair intermediate, HST mediate and VHIT the strongest acceleration.

\subsection{Main EVEST principles}

Data from Spontaneous Nystagmus (SPN), Caloric Test (CT), Head Shaking Test (HST), and Video Head Impulse Test (VHIT) were combined to estimate a frequency-specific hVOR characteristics.Clinicians carrying out vestibular tests are usually looking for asymmetries of semicircular canal function (especially lateral) between the two sides[9].All the tests within the EVEST can provide vestibular asymmetry evaluation. Despite this fact, we intentionally decided not to use the vestibular asymmetry as the only descriptive parameter.Vestibular asymmetry alone could not provide quick and clear overviewof the pathologies as bilateral vestibular loss, where vestibular asymmetry isoften low. VHIT is the only test wherethe absolute hVOR vestibular weakness of each ear can be evaluated. Therefore, we decided to use a mixture of results describing both, the vestibular asymmetry between both ears (SPN, CT, HST) and the absolute vestibular weakness of each ear separately (VHIT). Combination of both types of variables (vestibular asymmetry and absolute vestibular weakness) provides more thorough insight into the frequency specific changes of hVOR.

In three of the EVEST tests (SPN, CT, HST), whereprimarily vestibular asymmetry is observed,we plotthe weaker ear as a decreased $\mathrm{Y}$ value and the remaining ear as a zero value.This plotting principle shows a gap in the function between the weaker andthe remaining ear, which gap is represented asa relative functional loss in percentage. One could oppose that plotting the remaining ear as a zero value would not necessary 
represent the real state of its function, as both ears can be damaged to a different degree, or it could be also the hyperreflexive one.Observing the different degree of the vestibular function between both ears is handled precisely by the mixture of the asymmetry andthe weakness evaluation within the EVEST test results, as mentioned above.No doubt, the weaker ear could also be the healthy one,and the remaining ear could be the hyperreflexive one, but the EVEST resolves also this scenario, with plotting and highlighting the possible hyperreflexive caloric response to the table below the graph and by plotting the possible enhanced VHIT gain to the maximum negative value. Therefore, also the hyperreflexive ear is easily observed by visualization of the VOR function gap.

We emphasize that the vestibular weakness on the $Y$ axis, in case of the spontaneous nystagmus and the head shaking test, is not an absolute value, it is a visualization of the nystagmus intensity and an approximation of the vestibular asymmetry, based on our participant's data. Vestibular function, expressed on Y axis, serves as the graphic estimation of the vestibular function decrease/increase and provides follow up evaluation, as well as graphic evaluation of the static and dynamic VOR compensation. Scaling results of hVOR tests to an approximative vestibular weakness/asymmetryon the $\mathrm{Y}$ axis will be discussed further.

It's recommended that each department develop their own normative data.Nowadays there is still no agreement on normal and pathologic values of each hVOR test worldwide, as stressed byStrupp [10].

The rotatory chair, which is another hVOR dynamic test(tested VOR frequency $0.01-1 \mathrm{~Hz}$ ), could have been used in construction of the EVEST as well, but is not applied (none of hospital departments included in recent study owns the rotatory chair equipment). This limitation opens the possibility for improvement of EVEST for further studies.

\subsubsection{Spontaneous Nystagmus}

Nystagmus is an involuntary, rapid, rhythmic, oscillatory eye movement with at least one slow phase[11]. Jerk nystagmus is nystagmus with a slow phase and a fast phase. One of the first nystagmus classifications of the peripheral origin was established in 1912 by Alexander (Alexander's laws). In the scientific literature, few studies have focused on definition what is normal and what is abnormal, when speaking about spontaneous nystagmus(SPN) without fixation. The Barany Society made a consensus regarding the different nystagmus patterns[11].The criteria to differentiate normal against abnormal intensity and presenceof the spontaneous nystagmus in the healthy subjectsvary widely between the different laboratories. Recently, old criteria used for ENG have modified nowadays for VOG[1]. Kamran Barin (2006)gave a 95\% confidence interval of $4^{\circ} / \mathrm{s}[12]$, Levo et al (2004) found no horizontal PN among normal subjects[13], Svandelkova (2010) gave $3.2^{\circ} / \mathrm{s}[14]$, and Martens et al.(2016) gave $5^{\circ} / \mathrm{s}[15]$,Jeffery, Patel (2017) gave as low as [?]10/s[16], ANSI $>4 \mathrm{o} / \mathrm{s}[17]$. Vertical nystagmus withoutfixation has no currently-established guidelines for abnormality[17, 18]. Studies previously mentioned, evaluatepositional nystagmus in more than one position of head and head-neck-body position, and therefore have marginally different outcomes in detecting SPN than we do in our healthy group. Summarizing the literature, there is still a slight controversy and poor evidence of the presence of the spontaneous nystagmus in the single sitting upright position with the head still [19].

In our healthy group of 49participants none of them had any SPN on VOG tracing in a single upright sitting and head still position. According to our results, every nystagmus recorded with a head stillsitting in upright position, should be considered as a pathological finding. We tend to agree with studies that report no evidence of SPN in healthy subjects in upright sitting position, and if SPN was present, it should be considered as a probable vestibular "functional scar", as defined by Frenzel [19-24]. We concur with The British Society of Audiology's recommendation, published in 2015, in the absence of robust normative data, in case of any spontaneous or gaze-evoked nystagmus is present, with fixation removed, it needs to be interpreted in light of the overall pattern of test results and the patient's complaints[25]. For such recommendation the EVEST can serve as well. Any persistent repeatable nystagmus $\left(>1-2^{\circ} / \mathrm{s}\right)$ when in agreement with other significant test results (caloric weakness, rotating chair etc.) and a vestibular history, may be taken as a clear sign of pathology. It is always of most importance to determine whether the nystagmus follows Alexander's laws.

Based on our healthy group data,our reference value for SPN is $0^{\circ} / \mathrm{s}$ aSPV. Cutoff criteria should be based 
on mean $+-2 \mathrm{SD}$, what should be still 0 in our healthy group. Since the cutoff value has the same value as a reference one, our abnormalcutoff starts as far as slow phase velocity of $1^{\circ} / \mathrm{s}$ aSPV.Scale on the Y axis for spontaneous nystagmus is a nonlinear representation of the increasing slow phase velocity of the spontaneous nystagmus. We used the power function, approximative vestibular weakness asymmetry $[\%]=14 *$ aSPV ${ }^{\wedge} 1 / 2[\% / \mathrm{s}]$, which maps aSPV values of 0,15 to $50^{\circ} /$ sto approximative vestibular weakness of 0,54 and $99 \%$ respectively. The power function zoomed the first $15^{\circ} / \mathrm{s}$ aSPV of the SPN to a half of the Y axis range, which allows more detailed insight into theSPN changes within the first few weeksafter acute onset of vestibular loss. This composition of the SPN values on the Y axis allows also detailed insight into the regression of the nystagmusregarding to vestibular compensationas also as its time course(initially fast reduction of SPN intensity followed by the deceleratedreduction of SPN). We set up the nystagmus average slow phase velocity of $50^{\circ} /$ sas a maximum value in our scale (maximum in our neuronitis group was $31^{\circ} / \mathrm{s}$ aSPV, but we expect stronger nystagmus could be observed according to time course when the examination was done, so we set up a "pool" for even stronger nystagmus).Depending on the value set by individual labs, maximum value can be changed, and Y axis can be extended to even the higher values, if necessary. Similarly, depending on each vestibular lab consensus how to describe the characteristics of nystagmus, maximum slow phase velocity of spontaneous nystagmus instead of average slow phase velocity could be used in EVEST, and the same algorithm would be used in HST axis.

To summarize, the SPN vestibular weakness/asymmetryaxis,evaluates the intensity of the spontaneous nystagmus and serves as an approximative level of the vestibular function loss or function asymmetry.To express such a result, we use an approximation of vestibular weakness asymmetry in percentages.

\subsubsection{Plotting the results}

For plotting the SPN to the EVEST, we use average slow phase velocity (aSPV) [ $/ \mathrm{s}$ ] of detected nystagmus. Ifno spontaneous nystagmus is detected, both ears are plotted as a zero value. If any spontaneous nystagmus is present, we plot the aSPV [o/s] of measured horizontal SPN to the "SPN" Y axis. The ear, from which the spontaneous nystagmus is beating away, is the one with the relative vestibular weakness compared to the contralateral (remaining)one. This ear should be matched as a decreased $\mathrm{Y}$ value. The remaining ear should be plotted at the same time as a zero value on the $\mathrm{Y}$ axis, as we suppose interaural VOR gapruns thenystagmusactivity(due to asymmetry of the vestibular inputs from the peripheral vestibular endorgans). No doubt, the weaker ear could be also the healthy one, and stronger ear could be a hyperreflexive one, but it will be still plotted the same way (which means weaker is plotted as a decreased value, although could be a healthy one). From the literature evidence, this could happen f.e. in an early stage of the Meniere's attack, when irritative type of nystagmus hypothetically can be seen. The Y axis's vestibular weakness in "SPN" reflects intensity of SPN as well as the severity of vestibular function asymmetry.Also,in acute peripheral vestibular deafferentation (e.g. acute vestibular neuritis), as intensity of SPN decreases within first days to weeks, and therefore VOR gap decreases, the Y axis reflects a static compensation (reduction of vestibular asymmetry within the vetibular nucleai in the brainstem). SPN weakness asymmetry value also reflectsvestibular recoveryin the paroxysmal diseases. Up or down beating nystagmus alone or as a part of a horizontal nystagmus (also as detected torsion) $[0 / \mathrm{s}]$ is plotted into the table below the EVEST (see Figure 1).

\subsubsection{Caloric Test}

The caloric response was first described in 1906 byRobert Barany,who won a Nobel prize in 1914 for this discovery.It evaluates the degree to which the vestibular system is responsive and also how symmetric the caloric responses are, between left and right ears. Caloric test assesses and records the function of each LSCC separately. Procedure is based on the stimulation of the L-SCC by air (or water) induced temperature gradient. The thermal variationchanges the endolymph density within the stimulated L-SCC and produces theendolymph flow that deflects the cupula.

It is recommended that individual laboratories collect their own data on what constitutes the normal range of the caloric response[26].Alternatively, if normative data are not available, the normal limits of both canal 
paresis and directional preponderance may be taken as $+-20 \%[26]$.

\subsubsection{Plotting and interpreting the results}

For the EVEST, we use unilateral weakness (UW)resultas the principal resultto construct the EVEST.This result is worldwide routinely used when describing the caloric test result[26-29]. There are also directional preponderance (DP), totalresponse(TR), each irrigation response result, monothermal warm caloric screening test (MWST), that can be also used when describing the caloric test results and will be discussed further. In our healthy group, theunilateral caloric weakness was in almost all the subjects within a normal range $0-25 \%$ UW according to Jongkee's formula [28-30] (mean 12.7\%, SD 7.3\%, min 0, max 36).Abnormality cut-off in our healthy group was $27.3 \%$. The weaker ear is plotted as a decreased value on the $\mathrm{Y}$ axis, the remaining one isplotted as a zero value once again.

If UW is within the normal range, with a normal total caloric response, but monothermal warm caloric response (MWST) and/or DP is pathological, we should consider the MWST and/or DP results in context of remaining EVEST tests as well. For such purpose we have designed various symbols with written values of measured MWST or DP in a table under the EVEST (see Figure 1). In the case of pathological MWST result(more than $30 \%$ side difference)[31], the weaker ear is the one that is unmarked by the VOG software (f.e. MWST 30\%R means a 30\% stronger warm response on the right side, respectively the left ear was weaker during the warm caloric stimulation); for DP the dominated ear is associated with the measured stronger nystagmus direction (e.g.if DP $50 \% \mathrm{R}$ is present, the right ear seems to be the "stronger", respectively the left ear seems to bethe weaker). Pathological DP, in a normal total response range, reflects either warm caloric response difference (as MWST)due to peripheral vestibular endorgan pathology, or asymmetric vestibular nuclei function and internuclear projections (nystagmus beating stronger in one direction and evoked from the both sides, which means one ear is stronger in warm response, while the other ear is stronger in cold responce) $[32,33]$.

In presence of low total caloric response (in acute stage of peripheral vestibular loss with the presence of strong spontaneous nystagmus), DPreflects the inability to change the strong nystagmic activity by any of all the caloric stimuli (according to vestibular clamp).In aforementioned, in the VN groupin acute stage of vestibular loss, UW can be false negative, DP (which is usually about $80-100 \%$ ), should be used for the EVEST instead of UW (see Figure 3).

Further on, we need to consider the total caloric response to determine whether the UW is sufficient to describe the function of the peripheral vestibular endorgan. [34]. If the total response is less than $12^{\circ} / \mathrm{s}$ maximumSPV for cold and warm caloric responses, than it is unclear whether such a result is either a physiological (in our healthy 49 controls it was found in 5 cases with no history of vestibular pathology and normal results in additional vestibular tests) or a pathological one (i.e. bilateral hypo/areflexy). If the total response is low (although it could be a physiological response), the software usually does not calculate the UW and DP values, because they are not reliable. In the aforementioned cases, results should be always evaluated in accordancewith the other vestibular tests, for which the EVEST serves as well. In such case, both ears should be plotted into the EVEST with the same value of the vestibular loss on the Yaxis,or should not be plotted at all. Based on our experience such a case of a low total response, werecommendnot toplot anything intothe EVEST and only total response in $\% / \mathrm{s}$ should be provided in a table under the graph. The interpretation of results should be done according to presence or absence of spontaneous nystagmus and HST and VHIT results. If a suspicion of bilateral vestibular hyporeflexy is present, with total response below the $12^{\circ} / \mathrm{s}$ aSPV and L-SCC VHIT gains are $<0.5$ bilaterally, then pictograms for UW caloric test can be plotted asapproximately $80 \%-100 \%$ loss for both ears. If there is no evidence of any caloric response at all, pictograms for both ears can be marked as a $100 \%$ weakness. Westressthat every lab has to do its own normative caloric data. In our database from healthy volunteers, no caloric response was evaluated in 5 participants, even though no evidence of any cochleovestibular lesion was present. Caloric hyperreflexy (from our data-a response in any irrigation greater than $50^{\circ} / \mathrm{s} \mathrm{mSPV}$ )isprovided in the table under the graph.

\subsubsection{HST}


Head shaking nystagmus was first described in 1907 by Robert Barany, the first formal description of procedure was given later, in 1929, by Vogel. Headshaking-induced nystagmus is a jerk nystagmus that may follow a prolonged sinusoidal head oscillation[11], and lasting at least 5 seconds. Head-shaking nystagmus in the horizontal and vertical plane is abnormal. A welladjusted vestibular system should not produce headshakinginduced nystagmus, at least not for more than a second or so, because it reflects an unidirectional output for a sinusoidal input. This is abnormal and either means that the vestibular-ocular motor system is rectifying the input (clipping it in one direction), or that the system is being perturbed by the input. The main clinical utility of HSN to date has been to identify persons with unilateral peripheral vestibular lesions[1]. In normal subjects or persons with symmetrical vestibular loss (such as bilateral vestibular loss), no induced nystagmus is expected. In persons with a dynamic imbalance between the ears (such as due to unilateral vestibular neuritis or vestibular schwannoma), a nystagmus is often seen, usually beating towards the "better" ear, which decays over about 30 seconds[35]. In general, HSN should result from any vestibular asymmetry, whether it could be peripheral or central. It should always be interpreted in relation with other findings, such as spontaneous nystagmus and side of caloric weakness. The direction of head-shaking induced nystagmus may change during the course of recovery from a peripheral lesion.[25]

\subsubsection{Plotting the results}

For plotting the HST result into the EVEST, we use the aSPV results $\left[{ }^{\circ} / \mathrm{s}\right]$ from head shaking evoked nystagmus. Recently we did not find any recommendation for normative values and cut-off values in literature for HST induced nystagmus. In our healthy control group, none of healthy controls had head shaking induced nystagmus or even more than 1-2 nystagmic beats of low amplitude (1-2 $/ \mathrm{s} \mathrm{aSPV})$. If more than 3 clearly repetitive evoked nystagmus beats after the end of head shake are present, they should be analyzed.In the EVEST, plotting of HST uses the same rules as for plotting of SPN. From our vestibular schwannoma and neuritis group, decreased value of HST on the Y axis reflectsinitially static and afterwards the dynamicvestibular compensation.

\subsubsection{VHIT}

Head Impulse Test (HIT) was first described in 1988 by Halmagyi and Curthoys[36]. Implementation of aneye tracking system to the HIT started a new era of routineVideo Head Impulse testing (VHIT). The VHIT evaluates the functional state of the semicircular canalsby measuring the eye movement during an unpredictable head movement [8,36-39].In patients with unilateral vestibular lesion, eyes do not compensate for the ipsilesional head movement therefore slipped off the target. By the end of the head turn a corrective saccade occurs to return the gaze back to the target. Clinician should carefully evaluate the graphic results of the recording before putting any credence in the value of VOR gain. If the recordings are noisy, if there are large blinks or saccades (even before the peak head velocity) and/or if the eye velocity leads the head velocity, clinicians should be skeptical of the calculated VOR gain. [9]. Also, corrective saccades should be evaluated. The literature is unclear about the clinical value of interpretation of the corrective saccades. Curthoys suggests saccades to be a confirmation of the VHIT gains. The pattern of saccades can change even within a single testing session, but the VOR gain usually remains fixed, therefore VOR gain is important clinically primary indicator of vestibular function[9]. Contrary study tested the inter-examiners test results differencies on the same subjects and suggested saccades being more reliable parameter than a VHIT gain [40].

\subsubsection{Plotting the results}

Although all the remaining vestibular tests within the EVEST are describing the approximative vestibular weakness asymmetry, we decided not to use VHIT asymmetry(side difference)value [\%] of VOR gains from both sides, but the regression VOR gain value for each ear separately, as principal VHIT value to construct the EVEST. VOR gain asymmetry is alsocalculated [9] [\%] andprovided in the table below the EVEST.

Normative values for VHIT gains in our healthy group were calculated from mean VHIT gain +/-2SD (mean $0.87, \mathrm{SD} 0.04$ ). Cutoffs for our healthy group is 0.79- 0.95. When thinking about normal/abnormal value of the VHIT gain, there is also important to look for the saccades (overt or covert) and for the side difference. In 
the literature, there is still doubt about refferencevalues and cutoffs when assesing VHIT VOR gain[10, 41]. Each laboratory can use its normative data for VHIT gain cutoffs in the EVEST. Approximative vestibular weaknessof $0 \%$ equals the gain of 1.0 and the VHIT gain decrease of every 0.1 meansapproximative $10 \%$ vestibular function decrease (weakness increase).In contrast to other hVOR tests use for costruction ofthe EVEST, the VHIT is the only test, where results from both ears are plotted to the EVEST for each ear separately, and where EVEST does not only estimates an approximative asymmetry, but shows the real VOR gains for the each ear individually. The presence of the corrective overt and covert saccades is provided in the table below the EVEST.

\section{Conclusions}

To the best of our knowledge, we present a new and effective summarizing graph to visualizeand comparehVOR frequency-specific changes in different vestibular diagnoses.Multifrequency testing of hVOR is intended primarily to improve characterization of different degrees of peripheral vestibular deficit and to differentiate between different vestibular diagnoses. The EVESTservesas an estimation of the current vestibular function and if lesion is present, serves also for visualization of static and dynamic compensation.It isan easy-to-read graph, especially for otolaryngologists who are familiar with reading the audiograms which have similar basic principles, especially the visualization of functional decrease on the $\mathrm{Y}$ axis and frequency on the $\mathrm{X}$ axis.Plotting the both ears into the graph and graphic visualization of the depicted function decrease, allows EVEST to show the side difference between elucidated vestibular responses. This should allow a quickly understandingof the graph and the vestibular situation too.Clinicians carrying out vestibular tests are usually looking for asymmetries of semicircular canal function between the two sides.EVEST approximatesvestibular asymmetry and weakness. We tested feasibility of graphic visualization in different vestibular diagnoses with different levels of vestibular loss. Our construction and methodology of EVEST provides sufficient graphic overview of the vestibular dysfunction.

Some vestibular diagnoses show hVOR multifrequency involvement, such as an acute vestibular neuritis or labyrinthitisorcomplete vestibular loss, while otherscan involve only some hVOR frequencies. This dissociation in different hVOR tests is nowadays well documented in Meniere's disease as is in patients with vestibular schwannomas (also observed in our schwannoma group). In many patients with Meniere's disease, VHIT shows normal canal function, whereas caloric testing shows reduced (especially warm caloric response) or absent canal function. The similar situation can be observed in schwannoma patients, where the vestibular deficit isslowlyprogressing, and the high VOR frequencies (VHIT)become involved later than the low VOR frequencies (caloric test). All these mentioned situations the EVEST reflects very well.

Multifrequency testing of hVOR is essential for improved detection of any level of vestibular deficit within the L-SCC. It becomes even more apparent in cases with vestibular deficit slowly progressing over time and in the small steps, which provides the time for the continuous vestibular compensation and therefore poor clinical symptomatology, which might not be apparent at first sight, but with use of the EVEST becomes quite clear.

EVEST is able to clearly visualize all previously mentioned situations, what makes it easier for clinicians to interpret data from all the tests. In order to assist the researchers in clearly delivering their research data among different hVOR vestibular tests we can recommend to use cumulative EVEST graph for clear overview of researcher's conclusions. We recommend EVEST for clinicians as well as for educational purposes.

\section{References}

1. Hain, T.C., Head-shaking nystagmus and new technology.Neurology, 2007. 68 (17): p. 1333-4.

2. McGovern, T.N. and J.E. Fitzgerald, The effect of mental alerting on peripheral vestibular nystagmus during spontaneous, gaze (30 degrees left, 30 degrees right) and body positional (left 85 right lateral lying) testing using electronystagmography (ENG). Int J Audiol, 2008. 47 (10): p. 601-6.

3. Takahashi, J., K. Kitamura, and M. Miyata, Spontaneous nystagmus in normal subjects. ORL J Otorhinolaryngol Relat Spec, 1996.58 (1): p. 42-5. 
4. Humphriss, R.L., K.F. Morgan, and D.M. Baguley, The effect of mental alerting on gaze-evoked nystagmus. Clin Otolaryngol, 2005.30 (1): p. 25-8.

5. Koos, W.T., Criteria for preservation of vestibulocochlear nerve function during microsurgical removal of acoustic neurinomas.Acta Neurochir (Wien), 1988. 92 (1-4): p. 55-66.

6. Cohen, B. and T. Raphan, The Physiology of the Vestibuloocular Reflex (VOR), in The Vestibular System , S.M. Highstein, R.R. Fay, and A.N. Popper, Editors. 2004, Springer New York: New York, NY. p. 235-285.

7. Schmal, F., et al., The minimal ice water caloric test compared with established vestibular caloric test procedures. J Vestib Res, 2005. 15 (4): p. 215-24.

8. Halmagyi, G.M., et al., The Video Head Impulse Test. Front Neurol, 2017. 8 : p. 258.

9. Jacobson, G.P., et al., Balance Function Assessment and Management, Third Edition . 2020: Plural Publishing, Incorporated.

10. Strupp, M., et al., Worldwide survey on laboratory testing of vestibular function. Neurology: Clinical Practice, 2019: p. 10.1212/CPJ.0000000000000744.

11. Eggers, S.D.Z., et al., Classification of vestibular signs and examination techniques: Nystagmus and nystagmus-like movements. J Vestib Res, 2019. 29 (2-3): p. 57-87.

12. Kamran Barin, P., Common Errors in ENG/VNG. 2006.

13. Levo, H., H. Aalto, and T. Petteri Hirvonen, Nystagmus measured with video-oculography: methodological aspects and normative data. ORL J Otorhinolaryngol Relat Spec, 2004. 66 (3): p. 101-4.

14. Svadlenkova, I., Prevalence of positional nystagmus during static positional testing in normal healthy population using video-nystagmography. . 2010: p. 110p.

15. Martens, C., et al., Prevalence and Characteristics of Positional Nystagmus in Normal Subjects. Otolaryngol Head Neck Surg, 2016. 154 (5): p. 861-7.

16. Jeffery, H., et al., The interpretation of static positional nystagmus in a balance clinic. International Journal of Audiology, 2017. 56 (12): p. 958-966.

17. Kamran Barin, P.D., Best Practices in Administering VNG/ENG.2008.

18. Kamran Barin, P., Current State of Static Position Testing.2006.

19. Mulch, G. and W. Lewitzki, Spontaneous and positional nystagmus in healthy persons demonstrated only by electronystagmography: physiological spontaneous nystagmus or "functional scar"? Arch Otorhinolaryngol, 1977. 215 (2): p. 135-45.

20. Jatho, K., [Demonstration of latent vestibular spontaneous symptoms]. Arch Ohren Nasen Kehlkopfheilkd, 1958. 171 (2): p. 388-97.

21. Grohmann, R. and R. Meissner, [Evaluation of electronystagmographically registered spontaneous nystagmus in healthy persons]. Laryngol Rhinol Otol (Stuttg), 1983. 62 (11): p. 508-11.

22. Likhachev, S.A. and I.P. Mar'enko, [Spontaneous and provoked nystagmus in healthy subjects: on the so-called "vestibular" form/.Vestn Otorinolaringol, 2010(6): p. 21-5.

23. Jatho, K., /Vertigo/. Dtsch Med Wochenschr, 1977.102 (14): p. 537.

24. Mulch, G. and U. Trincker, [Physiological spontaneous and positional nystagmus. electronystagmographic examinations concerning type, frequency and intensity (author's transl)]. Laryngol Rhinol Otol (Stuttg), 1975. 54 (10): p. 841-53. 
25. Dr Ghada Al-Malky, M.J.B., Ms Katy Morgan, Ms Debbie Cane, Mr Albert Coelho, Mr Paul Radomskij, Dr Jennifer Rogers, Dr Peter West, Dr Andrew Wilkinson, British Society of Audiology, Recommended Procedure, Vestibular assessment - eye movement recordings. 2015.

26. Jacobson, G.P., Newman, C.W., \& Peterson, E.L. ,Interpretation and usefulness of caloric testing. 1993(Handbook of Balance Testing Function ): p. 193-233.

27. Jongkees, L.B.W., J.P.M. Maas, and A.J. Philipszoon, Clinical Nystagmography. Orl, 1962. 24 (2): p. 65-93.

28. Goncalves, D.U., L. Felipe, and T.M. Lima, Interpretation and use of caloric testing. Braz J Otorhinolaryngol, 2008. 74 (3): p. 440-6.

29. Murphy, K.A. and A.C. Anilkumar, Caloric Testing, inStatPearls . 2019: Treasure Island (FL).

30. Brookler, K.H., Importance of simultaneous binaural bithermal caloric testing. Ear Nose Throat J, 2002. 81 (4): p. 199.

31. Jacobson, G.P. and E.D. Means, Efficacy of a monothermal warm water caloric screening test. Ann Otol Rhinol Laryngol, 1985.94 (4 Pt 1): p. 377-81.

32. Cartwright, A.D., et al., Isolated directional preponderance of caloric nystagmus: II. A neural network model. Am J Otol, 2000.21 (4): p. 568-72.

33. Halmagyi, G.M., et al., Isolated directional preponderance of caloric nystagmus: I. Clinical significance. Am J Otol, 2000.21 (4): p. 559-67.

34. Aw, S.T., et al., Individual semicircular canal function in superior and inferior vestibular neuritis. Neurology, 2001.57 (5): p. 768-74.

35. Katsarkas, A., H. Smith, and H. Galiana, Head-shaking nystagmus (HSN): the theoretical explanation and the experimental proof. Acta Otolaryngol, 2000. 120 (2): p. 177-81.

36. Halmagyi, G.M. and I.S. Curthoys, A clinical sign of canal paresis. Arch Neurol, 1988. 45 (7): p. 737-9.

37. Curthoys, I.S., Generation of the quick phase of horizontal vestibular nystagmus. Exp Brain Res, 2002. 143 (4): p. 397-405.

38. Weber, K.P., et al., Impulsive testing of semicircular-canal function using video-oculography. Ann N Y Acad Sci, 2009.1164 : p. 486-91.

39. MacDougall, H.G., et al., The video head impulse test: diagnostic accuracy in peripheral vestibulopathy. Neurology, 2009.73 (14): p. 1134-41.

40. Korsager, L.E.H., et al., Reliability and comparison of gain values with occurrence of saccades in the EyeSeeCam video head impulse test (vHIT). Eur Arch Otorhinolaryngol, 2016. 273 (12): p. 4273-4279.

41. McGarvie, L.A., et al., The Video Head Impulse Test (vHIT) of Semicircular Canal Function - AgeDependent Normative Values of VOR Gain in Healthy Subjects. Front Neurol, 2015. 6 : p. 154.

\section{Appendix}




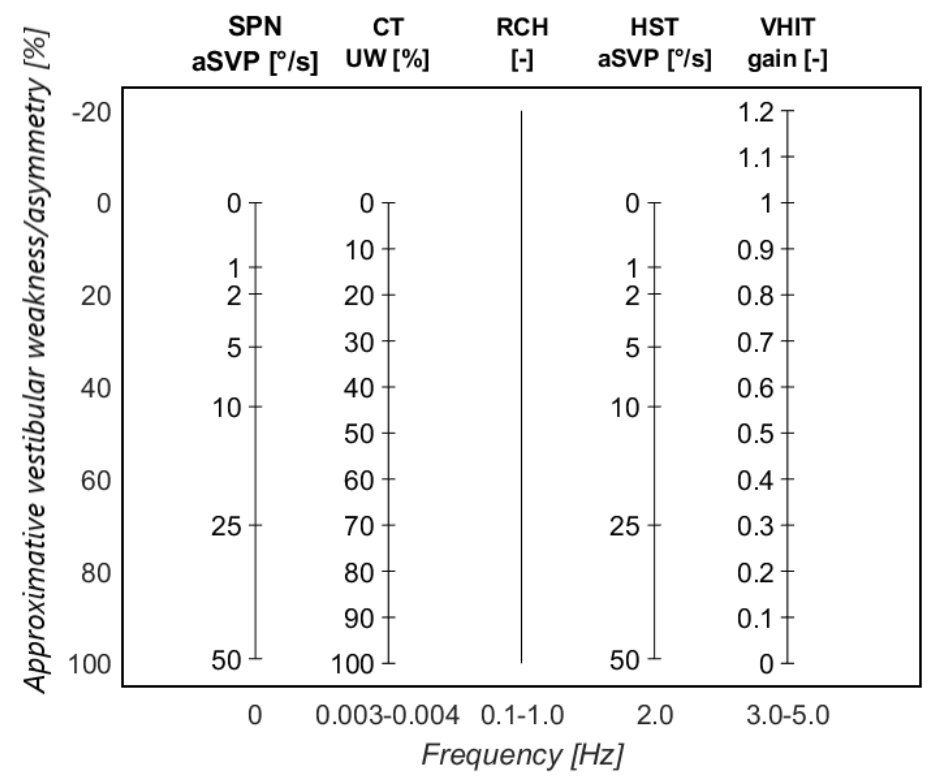

\section{Hosted file}

image2.emf available at https://authorea.com/users/319931/articles/449604-estimatedvestibulogram-evest-effective-graphic-tool-for-vestibular-assessment

FIGURE 1: Empty EVEST [SPN- Spontaneous Nystagmus, CT- Caloric Test, RCH- Rotatory Chair - not applied, HST- Head Shaking Test, VHIT- Video Head Impulse Test, aSPV - average slow phase velocity]

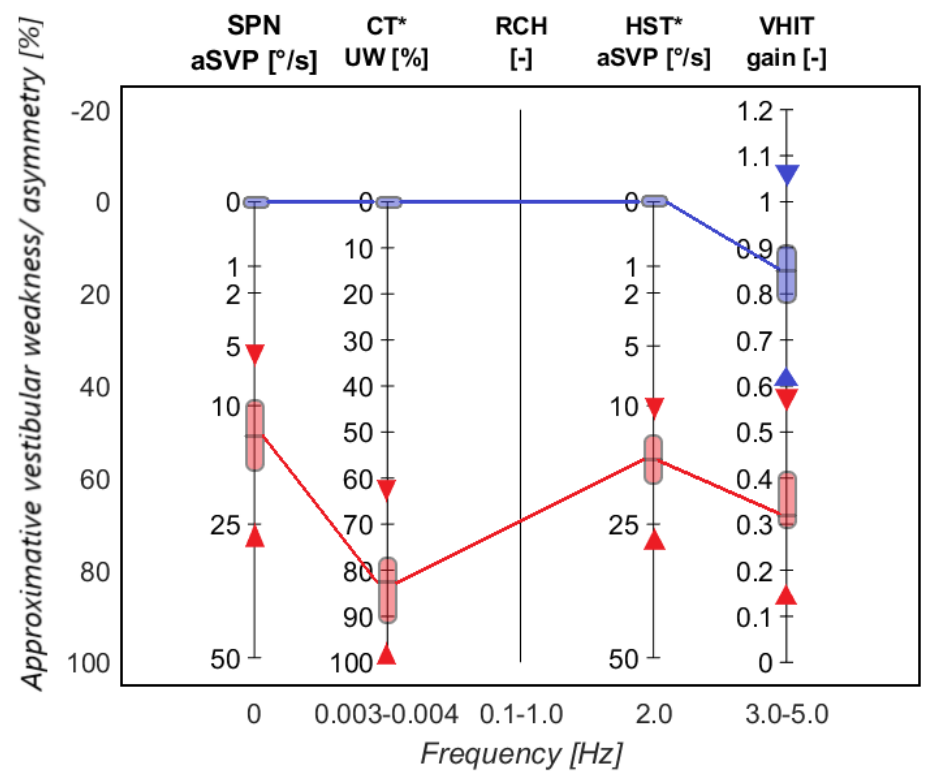


FIGURE 2: Cumulative EVEST for neuronitis group (for synoptical explanation are red (right) ears affected, remaining ears (healthy) are in blue). There is multifrequency involvement of all the vestibuloocular tests of the affected (red) ears within EVEST

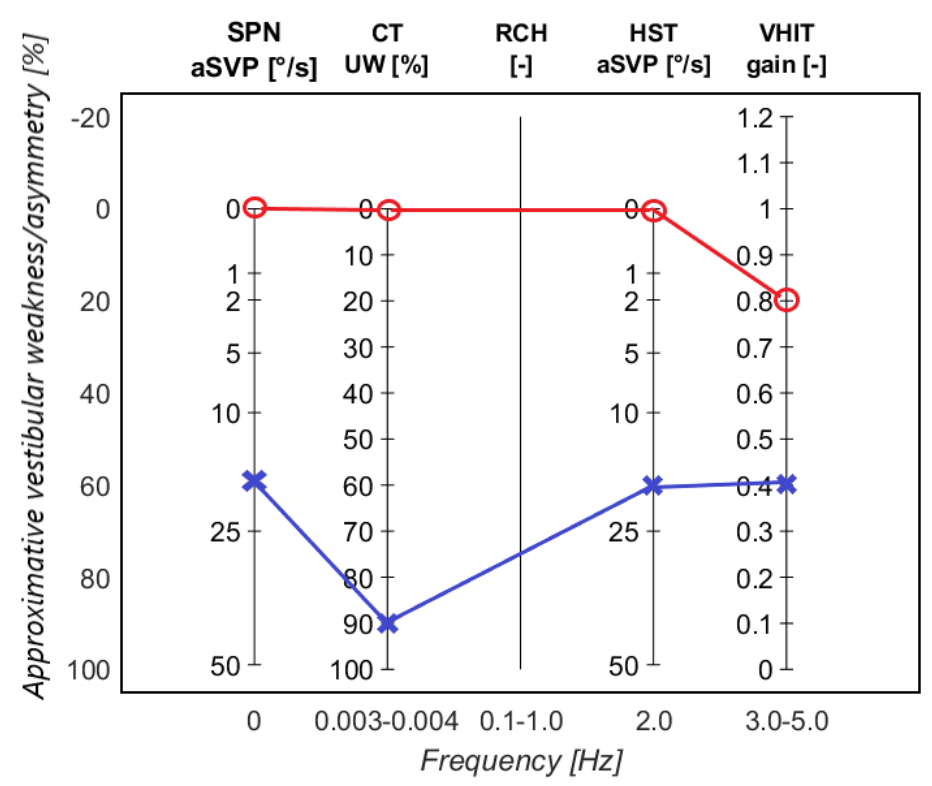

\section{Hosted file}

image5.emf available at https://authorea.com/users/319931/articles/449604-estimatedvestibulogram-evest-effective-graphic-tool-for-vestibular-assessment

FIGURE 3: Single EVEST of vestibular neuronitis patient in acute stage (also caloric test on the $3^{\text {rd }}$ day), the left side affected, multifrequency involvement of hVOR in acute vestibular neuronitis is clearly visualisated, instead of caloric unilateral weakness value, directional preponderance was used, no caloric stimulus could change the spontaneous nystagmus activity on the $3^{\text {rd }}$ day, UW was false negative, later confirmed on follow up after 3 months, when normal caloric response was on the left side andcaloric canal paresis on the affected side. 


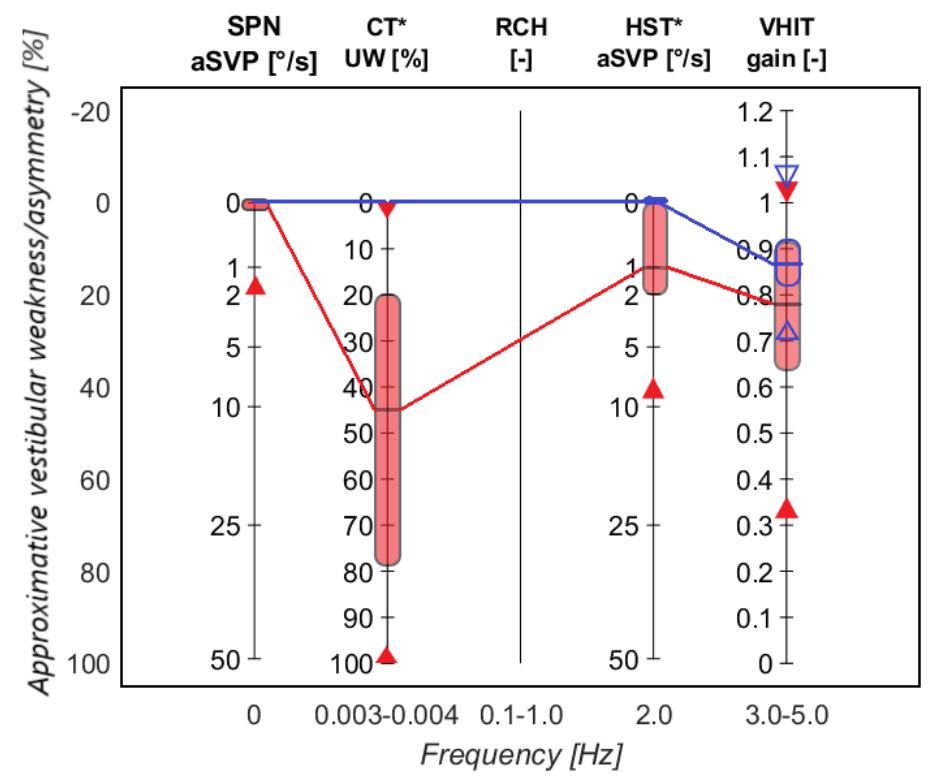

FIGURE 4: Cumulative EVEST for vestibular schwannoma group (the right (red) earsare like to be affected), also from cumulative EVEST can be clearly observed, that caloric test is mostly involved in all Koos schwannoma stages.

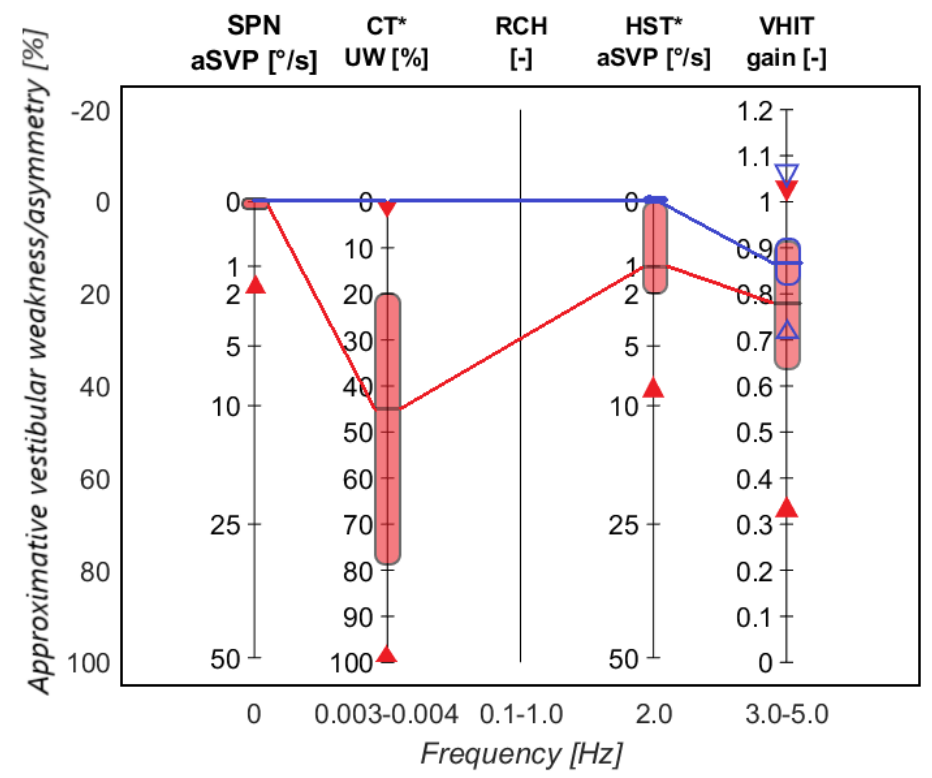

\section{Hosted file}

image8.emf available at https://authorea.com/users/319931/articles/449604-estimatedvestibulogram-evest-effective-graphic-tool-for-vestibular-assessment 
FIGURE 5: Single EVEST of the vestibular schwannoma patient, Koos 2 stage, affected is left side, the only test which shows the vestibular impairment is a caloric test (there were no vestibular complaints of the patient, it was incidental finding on an MRI, which was underwent because of the single-sided ringing tinnitus)

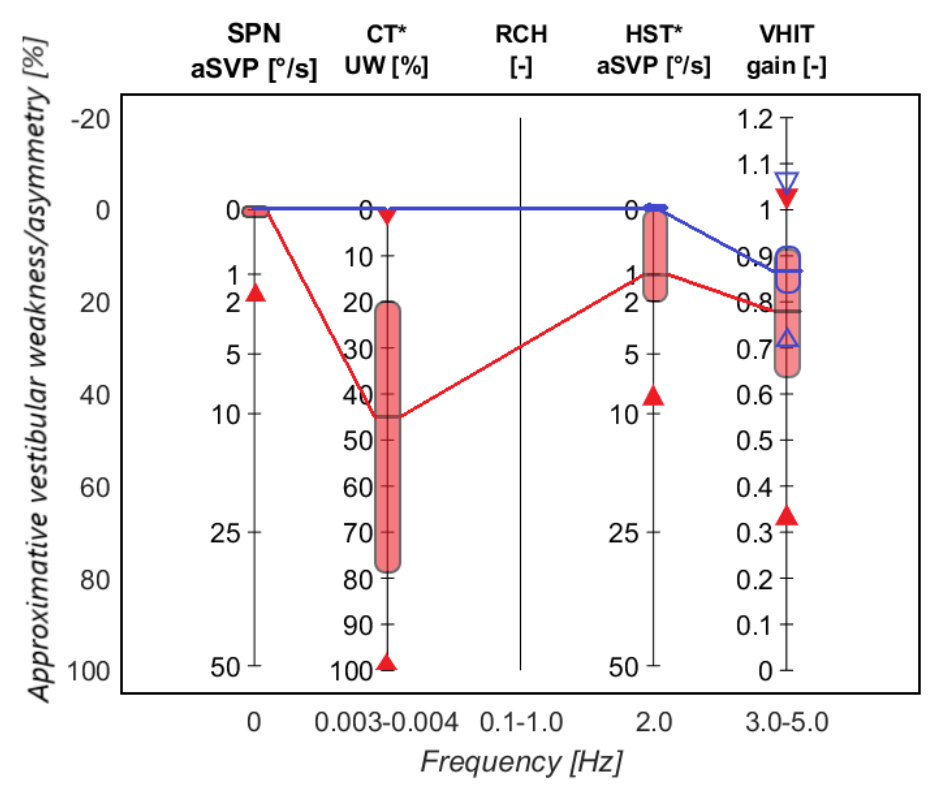

\section{Hosted file}

image10.emf available at https://authorea.com/users/319931/articles/449604-estimatedvestibulogram-evest-effective-graphic-tool-for-vestibular-assessment

FIGURE 6: Single EVEST, vestibular schwannoma l.sin., Koos 4 stage. Caloric test and VHIT show severe canal paresis. No spontaneous nystagmus is present, but induced head shaking nystagmus. 


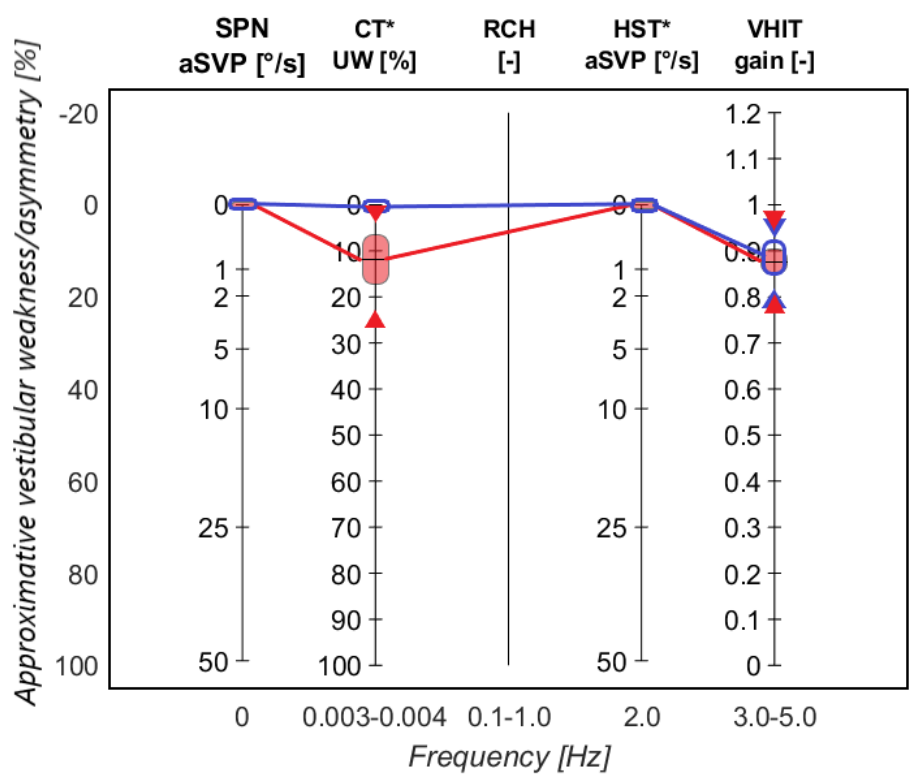

FIGURE 7: Cumulative EVEST for healthy volunteers group

\section{Hosted file}

fig 1.docx available at https://authorea.com/users/319931/articles/449604-estimatedvestibulogram-evest-effective-graphic-tool-for-vestibular-assessment

\section{Hosted file}

fig 2.docx available at https://authorea.com/users/319931/articles/449604-estimatedvestibulogram-evest-effective-graphic-tool-for-vestibular-assessment

\section{Hosted file}

fig 3.docx available at https://authorea.com/users/319931/articles/449604-estimatedvestibulogram-evest-effective-graphic-tool-for-vestibular-assessment

\section{Hosted file}

fig 4.docx available at https://authorea.com/users/319931/articles/449604-estimatedvestibulogram-evest-effective-graphic-tool-for-vestibular-assessment

\section{Hosted file}

fig 5.docx available at https://authorea.com/users/319931/articles/449604-estimatedvestibulogram-evest-effective-graphic-tool-for-vestibular-assessment

\section{Hosted file}

fig 6.docx available at https://authorea.com/users/319931/articles/449604-estimatedvestibulogram-evest-effective-graphic-tool-for-vestibular-assessment

\section{Hosted file}

fig 7.docx available at https://authorea.com/users/319931/articles/449604-estimatedvestibulogram-evest-effective-graphic-tool-for-vestibular-assessment 